\title{
Treatment of relapsed and refractory multiple myeloma
}

\author{
Ji Hyun Lee, Sung-Hyun Kim \\ Department of Internal Medicine, Dong-A University College of Medicine, Busan, Korea
}

p-ISSN 2287-979X / e-ISSN 2288-0011

https://doi.org/10.5045/br.2020.S008

Blood Res 2020;55:S43-S53.

Received on Novemver 25, 2019

Revised on May 13, 2020

Accepted on May 14, 2020

\begin{abstract}
The therapeutic strategy for relapsed and refractory multiple myeloma (RRMM) integrates a holistic approach regarding patient, disease, and drug-related factors. Patient-related factors include age, frailty status, and underlying comorbidities, especially cardiovascular and renal diseases and peripheral neuropathies that affect tolerability to multiple drug combinations or transplantations. Disease-related factors encompass these multiple patient-related factors, particularly the aggressiveness of the disease and cytogenetics. Regarding drug-related factors, the approval of novel proteasome inhibitors (such as carfilzomib and ixazomib), immunomodulatory agents (such as pomalidomide), monoclonal antibodies (such as daratumumab and elotuzumab), and new classes of drugs increasingly makes the choice treatment more complex and necessitates a comprehensive summary and an update of the efficacy and toxicities of each antimyeloma drug and its combinations. Further, careful monitoring of the side effects and supportive care throughout the course of treatment are important to achieve better outcomes for patients with RRMM.
\end{abstract}

Key Words Relapsed and refractory, Multiple myeloma, Treatment

\section{INTRODUCTION}

Survival for patients with multiple myeloma (MM) has markedly improved owing to recent progress in treatment strategies [1]. Nonetheless, MM remains incurable for most patients, and a significant proportion of patients with MM experience relapses that require further treatment. The introduction of next-generation immunomodulating agents (IMiDs), proteasome inhibitors (PIs), and monoclonal antibodies (mAbs) has widened treatment options; however, management of patients with relapsed and refractory MM (RRMM) requires a systematic approach. This review summarizes the published results of major clinical trials, as well as patient and disease-related factors, to help guide appropriate drug combinations and sequencing of therapy using currently available drugs.

\section{DEFINITIONS OF RELAPSE AND RELAPSED/REFRACTORY DISEASE}

Patients with RRMM present with three different disease patterns: 1) relapsed but not refractory, 2) relapsed and re- fractory, and; 3) primary refractory RRMM.

In 2008, the American Society of Hematology and the United States (US) Food and Drug Administration (FDA) Workshop established a uniform consensus on the definition of RRMM [2], and in 2016, the International Myeloma Working Group (IMWG) published a revised definition of relapsed $\mathrm{MM}$ [3].

\section{Relapsed disease}

Relapsed disease is defined as progressive disease after acquisition of a response to prior therapy that requires salvage therapy, but which does not meet the criteria for "primary refractory" or "relapsed and refractory" disease categories, based on laboratory and radiologic evidence, as follows:

\section{Biochemical relapse}

- $\geq 25 \%$ increase from the lowest confirmed response of the monoclonal protein (M-protein) in the serum (absolute increase, $\geq 0.5 \mathrm{~g} / \mathrm{dL}$ ) or in the urine (absolute increase, $\geq 200$ $\mathrm{mg} / \mathrm{d})$

- $\geq 25 \%$ increase from the lowest confirmed response between involved and uninvolved serum-free light chains (absolute increase, $>10 \mathrm{mg} / \mathrm{dL}$ )

- $\geq 10 \%$ increase of the absolute percentage of bone mar- 
row (BM) plasma cells

- New soft tissue plasmacytomas or bone lesions

- $\geq 50 \%$ (and $\geq 1 \mathrm{~cm}$ ) increase in existing plasmacytomas or bone lesions, as measured serially according to the sum of the products of the maximal perpendicular diameters (SPD) of the measured lesions

\section{Clinical relapse}

- Direct indicators of increasing disease and/or end organ dysfunction such as hypercalcemia, renal failure, anemia, and bone lesion (CRAB) features related to the underlying clonal plasma-cell proliferative disorder

- Serum calcium concentration $>11 \mathrm{mg} / \mathrm{dL}$

- Serum creatinine level $\geq 2 \mathrm{mg} / \mathrm{dL}$ (from the start of the therapy and attributable to myeloma)

- Decreased hemoglobin level by $\geq 2$ g/dL (not related to therapy or other non-myeloma-related conditions)

- Hyperviscosity related to serum paraprotein level

\section{Relapsed and refractory}

The term "relapse and refractory" designates disease in patients who achieve a minor response (MR) or better, and who then either become non-responsive while undergoing salvage therapy or who progress within 60 days of the last therapy.

\section{Primary refractory}

The term primary refractory designates refractory disease in patients who have never achieved an MR with any therapy. These include patients who never achieve an MR or better, for whom there is no significant change in the M-protein concentration and no evidence of clinical progression.

\section{DIAGNOSTIC APPROACH IN RELAPSE AND REFRACTORY MULTIPLE MYELOMA}

Several diagnostic procedures should be undertaken for patients with RRMM, including serum and urine protein electrophoresis and immunofixation, urine total protein, serum-free light chain, serum beta-2-microglobulin, and serum lactate dehydrogenase (LDH) tests. A peripheral blood smear test to detect circulating plasma cells is beneficial to discriminate high-risk patients. A bone marrow examination is mandatory, particularly for patients with non-secretory MM accompanied with fluorescent in situ hybridization (FISH) on monoclonal myeloma cells, and for patients who have not previously been identified with high-risk cytogenetics. Skeletal or extramedullary plasmacytoma evaluations using conventional $\mathrm{x}$-ray, computed tomography, magnetic resonance imaging, or positron emission tomography may be required for patients with suspected $\mathrm{MM}[4,5]$.

\section{SPECIFIC CONSIDERATIONS FOR TREATMENT OF PATIENTS WITH RRMM}

\section{Age and frailty}

The introduction of new agents has been reported to have prolonged survival in elderly patients [6]. Although age itself is not an obstacle for treatment, very elderly and frail patients are prone to experiencing treatment-related adverse events, leading to shorter survival [7]. Various frailty assessment tools, including the IMWG geriatric assessment tool, have been developed to predict outcomes concerning frail patients [8]. In one Korean study, scores used to predict poor overall survival (OS) for frail patients involved those related to age ( $\geq 80 \mathrm{yr}$ ), the Eastern Cooperative Group performance status (ECOG PS) 3-4, the estimated glomerular filtration rate (eGFR) $\left(<60 \mathrm{~mL} / \mathrm{min} / 1.73 \mathrm{~m}^{2}\right)$, and the presence of comorbidities ( $\geq 2$ comorbidities involving the heart, lung, or liver, cerebrovascular disease, and/or diabetes mellitus) [9]. The treatment goal for elderly and frail patients may focus more on symptom relief and the prevention of new myeloma-associated symptoms. Drug dose reductions and the selection of less intense regimens (such as doublet instead of triplet combinations) could be options more applicable to this patient group.

\section{Comorbidities}

Cardiac diseases (myocardial infarction, heart failure, and arrhythmias), renal impairment, peripheral neuropathy, diabetes mellitus, and thrombosis may be present at the time of MM diagnosis or as a consequence of previous antimyeloma therapies. Drugs likely to aggravate underlying diseases or residual toxicities that require monitoring for organ-specific complications are as follows: anthracyclines and carfilzomib (cardiotoxicities); lenalidomide and ixazomib, which require dose reductions for patients with impaired renal function; thalidomide, bortezomib, and vincristine (peripheral neuropathy); corticosteroids (glucose intolerance), and; IMiDs (thrombosis).

\section{Aggressiveness at relapse}

Several parameters have been proposed as poor prognostic factors in RRMM. Extramedullary disease has been observed in approximately $14 \%$ of patients at relapse of MM and is usually associated with high-risk cytogenetic abnormalities affecting poor OS [10, 11]. An elevated LDH level [12], a peripheral blood plasma cell count that does not fulfill the criteria for plasma cell leukemia (a peripheral blood plasma cell count of $>20 \%$ of the total white blood cell count, and an absolute count of $\geq 2,000 / \mu \mathrm{L}$ ) [13], and a high plasma cell proliferative index at post-autologous stem-cell transplantation (post-auto-SCT) D+100 [14] are indicators for short survival. Several studies have recommended that the rapid onset of disease-related organ damage (hypercalcemia, renal failure, and bony complications), as well as the above listed factors predictive of rapid disease progression, be defined as "aggressive disease" that requires 
treatment intervention using the most effective regimens $[4,5,15]$.

\section{Cytogenetics}

The effect of high-risk cytogenetics defined using del(17p), $\mathrm{t}(4 ; 14)$, and $\mathrm{t}(14 ; 16)$ on the aggressive disease evolution is not straightforward. Different studies involving salvage lenalidomide-dexamethasone ( $\mathrm{Rd}$ ) have reported controversial outcomes regarding $\operatorname{del}(17 \mathrm{p})$ and $\mathrm{t}(4 ; 14)[16,17]$. Some of the currently available combination therapies, such as pomalidomide-dexamethasone (Pd, MM-003) [17], carfilzomibdexamethasone (Kd, ENDEAVOR) [18], ixazomib-Rd (IRd, TOURMALINE MM-1) [19], and daratumumab-bortezomibdexamethasone (Dara-Vd) (CASTOR) [20], have significantly improved the poor outcomes of high-risk disease. Further, studies on Rd with or without carfilzomib (ASPIRE) [21], elotuzumab (ELOQUENT-2) [22], and daratumumab (POLLUX) [23] have reported that these drugs improve pro- gression-free survival (PFS) but not significantly. A summary of each trial concerning these high-risk cytogenetics affecting PFS is listed in Table. However, these results should not be compared directly due to the inconsistency of FISH methods and cut-offs in the different studies. Results of the major clinical trials in the experimental arm and in the high-risk cytogenetics group are summarized in Tables 1 and 2 .

\section{OPTIONS FOR ANTIMYELOMA TREATMENT IN RRMM}

\section{Treatment indications and timing of therapy}

The objective of treatment in the relapse setting is to alleviate symptoms, prevent progressive organ damage, and re-attain long-lasting disease remission. Indications to initiate therapy are clinical relapse, defined according to the CRAB criteria, or a significant increase of biochemical relapse in 2 consecutive measurements within 2 months apart that

Table 1. A summary of selected phase II and III clinical trials.

\begin{tabular}{|c|c|c|c|c|c|c|c|c|}
\hline Study & $\begin{array}{l}\text { POLLUX } \\
{[23,58]}\end{array}$ & $\begin{array}{l}\text { ASPIRE } \\
{[33,34]}\end{array}$ & $\begin{array}{c}\text { ELOQUENT-2 } \\
{[22,62]}\end{array}$ & $\begin{array}{l}\text { TOURMALIN } \\
\text { E MM-1 [19] }\end{array}$ & $\begin{array}{l}\text { CASTOR } \\
{[20,59]}\end{array}$ & $\begin{array}{c}\text { ENDEAVOR } \\
{[18]}\end{array}$ & $\begin{array}{c}\text { MM-003 } \\
{[17]}\end{array}$ & $\begin{array}{c}\text { GEN501 and } \\
\text { SIRIUS [54] }\end{array}$ \\
\hline Regimen & DRd (vs. Rd) & KRd (vs. Rd) & ERd (vs. Rd) & IRd (vs. Rd) & DVd (vs. Vd) & $\mathrm{Kd}$ (vs. Vd) & Pd (vs. D) & Daratumumab \\
\hline $\mathrm{N}$ & 569 & 792 & 646 & 722 & 498 & 929 & 302 & 148 \\
\hline Median prior lines (range) & $1(1-11)$ & $2(1-3)$ & $2(1-4)$ & $1-3$ & $2(1-9)$ & $2(1-2)$ & $5(2-14)$ & 5 \\
\hline ORR ( $\geq$ VGPR, \%) & $92.9(75.8)$ & 87 (69.9) & $79(35)$ & $72(48)$ & $83.8(62.1)$ & $77(54)$ & $31(6)$ & $31.1(13.5)$ \\
\hline PFS (HR, mo) & $\begin{array}{l}\mathrm{N} / \mathrm{R} \text { at } \\
25.4 \mathrm{mo} \\
(\mathrm{HR}, 0.41)\end{array}$ & $\begin{array}{l}26.3 \\
(\mathrm{HR}, 0.69)\end{array}$ & $\begin{array}{l}19.4 \\
(H R, 0.70)\end{array}$ & $\begin{array}{l}20.6 \\
(\mathrm{HR}, 0.74)\end{array}$ & $\begin{array}{l}16.7 \text { at } \\
19.4 \text { mo } \\
(\mathrm{HR}, 0.31)\end{array}$ & $\begin{array}{l}18.7 \\
(\mathrm{HR}, 0.53)\end{array}$ & $\begin{array}{l}4.0 \\
(H R, 0.48)\end{array}$ & 4.0 \\
\hline OS (HR, mo) & $\begin{array}{c}92.1 \% \text { at } \\
12 \mathrm{mo}\end{array}$ & $\begin{array}{l}48.3 \\
(\mathrm{HR}, 0.79)\end{array}$ & $\begin{array}{c}48(\mathrm{HR}, \\
0.78)\end{array}$ & $\mathrm{N} / \mathrm{A}$ & $\mathrm{N} / \mathrm{A}$ & $\begin{array}{l}47.6 \\
(\mathrm{HR}, 0.79)\end{array}$ & $\begin{array}{l}11.9 \\
(H R, 0.53)\end{array}$ & 20.1 \\
\hline
\end{tabular}

Abbreviations: D, high-dose dexamethasone; DRd, daratumumab-Rd; DVd, daratumumab-Vd; ERd, elotuzumab-Rd; HR, hazard ratio; IRd, ixazomib-Rd; Kd, carfilzomib-dexamethasone; KRd, carfilzomib-Rd; N, number of patients; N/A, not available; N/R, not reached; ORR, overall response rate; OS, overall survival; Pd, pomalidomide-dexamethasone; PFS, progression-free survival; Rd, lenalidomide-low-dose dexamethasone; Vd, bortezomib-dexamethasone; VGPR, very good partial response.

Table 2. The efficacy of triplet and doublet combinations in patients with high-risk cytogenetics.

\begin{tabular}{|c|c|c|c|c|c|}
\hline & \multirow{2}{*}{ Regimen } & \multirow{2}{*}{$\begin{array}{c}\text { High risk } \\
\text { cytogenetics (\%) }\end{array}$} & \multicolumn{3}{|c|}{ Median PFS $(\mathrm{HR}, P)$} \\
\hline & & & All high-risk & $\operatorname{del}(17 p)$ & $t(4 ; 14)$ \\
\hline POLLUX [58] & DRd vs. Rd & $15.4 \%$ vs. $16.6 \%$ & $\begin{array}{l}22.6 \text { vs. } 10.2 \mathrm{mo} \\
(\mathrm{HR}, 0.53 P=0.921)\end{array}$ & $\mathrm{N} / \mathrm{A}$ & NA \\
\hline ASPIRE [21] & KRd vs. Rd & $12.1 \%$ vs. $13.1 \%$ & $\begin{array}{l}23.1 \text { vs. } 13.9 \mathrm{mo} \\
(\mathrm{HR}, 0.70 P=0.0829)\end{array}$ & $\begin{array}{l}24.5 \text { vs. } 11.1 \mathrm{mo} \\
(\mathrm{HR}, \mathrm{N} / \mathrm{A})\end{array}$ & $\begin{array}{l}23.1 \text { vs. } 16.7 \mathrm{mo} \\
(\mathrm{HR}, \mathrm{N} / \mathrm{A})\end{array}$ \\
\hline ELOQUENT-2 [22, 62] & ERd vs. Rd & $\mathrm{N} / \mathrm{A}$ & $\mathrm{N} / \mathrm{A}$ & $\begin{array}{l}21.2 \text { vs. } 14.9 \mathrm{mo} \\
(\mathrm{HR}, 0.65)\end{array}$ & $\begin{array}{l}15.8 \text { vs. } 5.5 \text { mo } \\
(H R, 0.53)\end{array}$ \\
\hline TOURMALINE MM-1 [19] & IRd vs. Rd & $21 \%$ vs. $17 \%$ & $\begin{array}{l}21.4 \text { vs. } 9.7 \mathrm{mo} \\
\quad(\mathrm{HR}, 0.543 P=0.021)\end{array}$ & 21.4 vs. $9.7(H R, 0.596)$ & $\begin{array}{l}18.5 \text { vs. } 12 \mathrm{mo} \\
(\mathrm{HR}, 0.645)\end{array}$ \\
\hline CASTOR $[20,59]$ & DVd vs. Vd & $22.7 \%$ vs. $21.3 \%$ & $\begin{array}{l}11.2 \text { vs. } 7.2 \mathrm{mo} \\
(\mathrm{HR}, 0.45 P=0.0053)\end{array}$ & N/A & NA \\
\hline ENDEAVOR [18] & Kd vs. Vd & $21 \%$ vs. $24 \%$ & $\begin{array}{l}8.8 \text { vs. } 6.0 \mathrm{mo} \\
\quad(\mathrm{HR}, 0.646 P=0.025)\end{array}$ & $\begin{array}{l}7.6 \text { vs. } 4.9 \mathrm{mo} \\
(\mathrm{HR}, \mathrm{N} / \mathrm{A} P=0.13)\end{array}$ & $\begin{array}{l}10.1 \text { vs. } 6.8 \mathrm{mo} \\
(\mathrm{HR}, \mathrm{N} / \mathrm{A} P=0.03)\end{array}$ \\
\hline
\end{tabular}

Abbreviations: DRd, daratumumab-Rd; DVd, daratumumab-Vd; ERd, elotuzumab-Rd; HR, hazard ratio; IRd, ixazomib-Rd; Kd, carfilzomibdexamethasone; KRd, carfilzomib-Rd; N/A, not available; OS, overall survival; PFS, progression-free survival; Rd, lenalidomide-low-dose dexamethasone; $\mathrm{Vd}$, bortezomib-dexamethasone. 
meets the IMWG criteria for the definition of progressive disease [5]. Relapses with acute onset and rapid progression of symptoms require a prompt initiation of treatment.

Asymptomatic biochemical relapses could be monitored every 1-2 months with careful observation of symptoms. However, for high-risk disease features indicative of a poor response and a short OS, such as "aggressive disease" at relapse, a treatment-free interval of $<12$ months with a suboptimal response to prior therapy and unfavorable risk cytogenetics $[\mathrm{t}(4 ; 14), \mathrm{t}(14 ; 16)$, and $\operatorname{del}(17 \mathrm{p})]$, treatment should be started immediately. A recent subgroup analysis of patients in the ENDEAVOR study, which compared the results of asymptomatic biochemical and symptomatic relapse in $\mathrm{Kd}$ and Vd groups, demonstrated a better median PFS and OS in patients who commenced treatment at the time of asymptomatic biochemical relapse rather than at clinical relapse in both groups [24]. This result indicates that therapeutic intervention at biochemical relapse before (re)appearance of clinical symptoms might be of benefit to patients in terms of PFS and OS.

\section{Treatment options for RRMM}

Retreatment: When patients with RRMM demonstrate indications for treatment, changing the class of available drug is preferable. However, retreatment involving previously prescribed drugs is an acceptable option if the previous therapy achieved at least a partial response (PR) and if that response was maintained for at least 6 months with an acceptable toxicity profile. Prospective registry data indicate that bortezomib retreatment is a feasible option, with a response rate of $46 \%$, including $15 \%$ complete response (CR)/nearCR (nCR), with a median PFS and OS from the start of retreatment of 6.4 months and 17.6 months, respectively. Peripheral neuropathy during bortezomib retreatment occurred in $49 \%$ of patients, including $10 \%$ of those with grade $3 / 4$ toxicity [25]. Repeated use of IMiDs is also a feasible option with an overall response rate (ORR) of $44 \%$. The response rate of lenalidomide retreatment in previous lenalidomide responders has been reported to be $58 \%$ in one study [26]. Prior to retreatment, remaining toxicities due to previous therapy such as peripheral neuropathies should be considered. Reuse of previously prescribed drugs may require a less intense dosage and a change to administration schedules.

\section{Proteasome inhibitors}

Bortezomib: Bortezomib monotherapy is more effective in patients with RRMM than high-dose dexamethasone, with a better ORR (38\% vs. 18\%) reported in the phase III APEX trial [27], a better time-to-progression (TTP, 6.2 vs. $3.5 \mathrm{mo}$ ), and a 1 -year OS (80\% vs. $66 \%)$. Bortezomib is also active in patients with poor renal function with an acceptable toxicity profile. Peripheral neuropathy is a well-known adverse event of bortezomib treatment and its incidence can be reduced through subcutaneous administration [28]. For elderly patients, lower dose-intensity regimens (e.g., a weekly administration) have been reported to improve adherence to therapy in this vulnerable patient group [29]. Several combi- nation regimens with $\mathrm{Vd}$ have shown improved efficacies with manageable toxicity profiles. Pegylated liposomal doxorubicin with bortezomib significantly improved PFS (9.3 vs. $6.5 \mathrm{mo})$ and duration of response (10.2 vs. $7.0 \mathrm{mo})$ compared with bortezomib monotherapy in a phase III international trial [30]. In previous autologous stem cell transplantation (autoSCT) recipients, a triple combination of thalidomide and Vd compared with thalidomide and dexamethasone demonstrated a significantly better PFS (19.5 vs. $13.8 \mathrm{mo}$ ) and $\mathrm{nCR} / \mathrm{CR}$ rate ( $45 \%$ vs. $21 \%$ ), but also showed higher grade 3 and 4 neurotoxicity (MMVAR/IFM 2005-04) [31]. Vd has also been used as a backbone for combinations with new classes of drugs, such as daratumumab (Dara-Vd) and panobinostat (Pano-Vd).

Carfilzomib:The intravenous second-generation PI, carfilzomib, irreversibly binds to the chymotrypsin-like site of proteasome and its major toxicities involve cardiovascular complications such as heart failure, hypertension, and ischemic heart disease. In patients with RRMM who received a median of 5 prior regimens, carfilzomib as a single agent was not superior to low-dose corticosteroids with or without cyclophosphamide in terms of OS and PFS, indicating that carfilzomib should be administered in combination with other drugs, and in the earlier lines of chemotherapy (FOCUS trial) [32]. In 2015, carfilzomib plus Rd (KRd) showed markedly improved PFS (26.3 vs. $17.6 \mathrm{mo}$ ) and ORR (87\% vs. $67 \%$ ) (ASPIRE trial) [33]. The final survival analysis at a median follow-up of 67 months showed significantly better OS in the KRd group (48.3 vs. 40.4 mo) [34]. The KRd regimen showed favorable outcomes across age subgroups [35]. Kd also significantly improved PFS (18.7 vs. $9.4 \mathrm{mo}$ ), OS (47.6 vs. $40 \mathrm{mo})$, and ORR (77\% vs. 62\%) compared with Vd in the ENDEAVOR trial [36]. The published results of a recent interim analysis of the phase III A.R.R.O.W. trial compared once-weekly $\left(20 \mathrm{mg} / \mathrm{m}^{2}\right.$ day 1 during cycle 1 ; and $70 \mathrm{mg} / \mathrm{m}^{2}$ thereafter), twice-weekly $\left[20 \mathrm{mg} / \mathrm{m}^{2}\right.$ days 1 and 2 (cycle 1); and $27 \mathrm{mg} / \mathrm{m}^{2}$ thereafter] doses of carfilzomib [37]. Patients in the once-weekly group showed better outcomes for PFS (11.2 vs. $7.6 \mathrm{mo}$ ) and ORR (62.9\% vs. $40.8 \%$ ) with similar safety profiles in both dosage arms. Subgroup analysis of Asian patients participating in the ENDEAVOR and A.R.R.O.W trials showed a comparable efficacy but increased (grade 3 or higher) cardiovascular toxicities in the carfilzomib treated group [38].

Ixazomib: Ixazomib is an oral next-generation boron-based PI. In the TOURMALINE-MM1 phase III trial, the ixazomib plus Rd (IRd) group showed a significantly superior PFS (20.6 vs. $14.7 \mathrm{mo}$ ) and ORR (78\% vs. $72 \%$ ) than the $\mathrm{Rd}$ control group [19]. Notably, the efficacy of IRd was significant in high-risk cytogenetic risk groups (HR, 0.54; $P=0.02$ ). The toxicity profile was comparable in both study groups, although an ixazomib combination regimen showed a slightly higher rate of skin rash and peripheral neuropathy. A phase II/III study involving ixazomib-dexamethasone in combination with pomalidomide in patients with RRMM is currently ongoing (NCT 03170882). Novel PIs (oprozomib and marizomib) as single or combination chemotherapies 
have also shown promising efficacies in phase I studies [39-41].

\section{IMiDs}

Thalidomide: Thalidomide, the first approved IMiD, showed a response rate of $25-55 \%$ in patients with RRMM as a monotherapy. Using a $\geq 2$-drug combination with dexamethasone, bortezomib, lenalidomide, pegylated liposomal doxorubicin, or alkylating agents, the ORR improved from $63 \%$ to $90 \%$, with a CR rate of between $2 \%$ and $36 \%$. Retreatment with thalidomide among previous thalidomide responders has been reported to be $40 \%$ [26]. A multicenter, real-world data study involving 367 patients with RRMM treated with either a thalidomide, cyclophosphamide, and dexamethasone (CTD) regimen or a thalidomide, melphalan, and prednisolone (MTP) regimen reported an ORR of $72 \%$ versus $65 \%$, respectively, which was not significantly different. A combination regimen such as thalidomide, alkylating agent, and corticosteroid (TAS) showed a median PFS and OS of 10.4 months and 28.0 months, respectively. However, $10 \%$ of patients experienced severe infectious complications that warranted intensive supportive care during the TAS treatment [42]. While the use of thalidomide in the RRMM setting is now less frequent due to the advent of more potent drugs and due to its considerable side effects, such as peripheral neuropathy, fatigue, and venous thromboembolism, thalidomide plus corticosteroid therapy with or without an alkylating agent could be an option in specific circumstances.

Lenalidomide:Lenalidomide is a second-generation IMiD with less peripheral neuropathy but greater BM toxicity than thalidomide. It has been proven to be active as a single agent in RRMM, with an ORR of $25-27 \%$ and a median OS of 23-27 months. From the pooled analysis of two phase III MM-009 and MM-010 trials after 48 months of follow-up, heavily pretreated patients with RRMM who received lenalidomide $25 \mathrm{mg}$ in combination with dexamethasone (Rd) showed a significantly better ORR (60.6\% vs. $21.9 \%)$, CR (15.0\% vs. $2.0 \%$ ), TTP (13.4 vs. $4.6 \mathrm{mo}$ ), and OS (38.0 vs. $31.6 \mathrm{mo}$ ) compared with a dexamethasone-only group [43, 44]. Korean real-world experience of Rd showed an ORR of $43.6 \%$, with $15.4 \%$ of patients showing a very good partial response or better, a median TTP, and an OS of 8 and 23 months [45]. A phase II trial involving a bortezomib, lenalidomide, and dexamethasone (VRd) regimen reported an ORR of $64 \%$ and a median PFS of 9.5 months in patients with RRMM [46]. Currently, triplet regimens using Rd in combination with carfilzomib (KRd), ixazomib (IRd), daratumumab (Dara-Rd), and elotuzumab (Elo-Rd) have shown better efficacy in phase III trials.

Pomalidomide: In a phase III MM-003 trial involving patients with RRMM who failed to respond to both bortezomib and lenalidomide, pomalidomide with low dose dexamethasone $(\mathrm{Pd})$ proved to have superior efficacy compared with dexamethasone alone in terms of ORR (31\% vs. $10 \%)$, PFS (4.0 vs. $1.9 \mathrm{mo})$, and OS (12.7 vs. $8.1 \mathrm{mo}$ ) [47]. The major side effect of Pd was myelosuppression and neutropenic fever. Among the high-risk cytogenetic abnormal- ities, del(17p) appeared to benefit from pomalidomide treatment (IFM 2010-02 trial) [48]. Cyclophosphamide plus Pd (PCd) is an option for lenalidomide-refractory heavily pretreated patients with MM, with a better ORR $(64.7 \%$ vs. $38.9 \%$ ) and PFS (9.5 vs. $4.4 \mathrm{mo}$ ) than Pd [49]. A real-world study conducted by the Korean Multiple Myeloma Working Group involving a Kd, Pd, and PCd regimen showed a better PFS in a PCd treated group compared with doublet combination chemotherapies in patients who had relapsed or who were refractory to 2 or more lines of therapies [50]. In the recent phase III OPTIMISSM trial, pomalidomide in combination with $\mathrm{Vd}(\mathrm{PVd})$ also demonstrated a better ORR $(82.2 \%$ vs. $50 \%$ ) and PFS (11.2 vs. $7.1 \mathrm{mo}$ ) than $\mathrm{Vd}$, even in the lenalidomide-refractory subgroup [51].

\section{Monoclonal antibodies}

Daratumumab:Daratumumab (Dara) is an mAb-targeting CD38 surface antigen on MM cells. Its clinical efficacy as a monotherapy for patients with RRMM has been studied in the phase I/II GEN501 study [52] and the results have been confirmed in the phase II SIRIUS study [53]. A report on the combination of part 2 of the GEN501 and SIRIUS studies that included patients who had received a median of 5 prior lines of therapies showed an ORR of $31.1 \%$, a median duration response of 7.6 months, a PFS of 4.0 months, and an OS of 20.1 months [54]. In the final results of the GEN501 and SIRIUS studies, the 3-year PFS was 19.6\%, showing maintenance of a deep and durable response [55]. Approximately $50 \%$ of the patients were found to have experienced infusion-related reactions (IRR) during Dara administration, most of which occurred in the first infusion and were categorized as either grades 1 or 2 . In a real-world study involving patients from Korean medical centers, the ORR and PFS in relation to Dara monotherapy was reported to be from $42.1 \%$ to $56.3 \%$ and from 2.7 to 6 months, respectively, with any grade IRR from $42.9 \%$ to $87.5 \%$, which were mostly manageable $[56,57]$.

Phase III clinical trials evaluating Dara in combination with a fixed duration of Vd (CASTOR) [20] and with continuous Rd (POLLUX) [23] have proven their synergistic efficacy compared with a comparator arm. The POLLUX trial reported prolonged PFS (median not reached vs. 17.5 mo) and a better ORR ( $92.9 \%$ vs. $76.4 \%)$ in the Dara-Rd arm compared with $\mathrm{Rd}$ at 25.4 months follow-up. A PFS benefit in the Dara-Rd arm was observed across age subgroups and ISS stage [58]. In the 19.4 months of updated analysis from the CASTOR trial, 8 cycles of Dara-Vd followed by Dara maintenance improved PFS (16.7 vs. $7.1 \mathrm{mo}$ ) and ORR (83.8\% vs. $63.2 \%)$ compared with Vd only. Dara-Vd was effective regardless of age, ISS stage, renal failure, and cytogenetic risk [59]. In the POLLUX and CASTOR trials, a significant rate of MRD negativity was achieved across both high and standard cytogenetic risk patients [60]. Phase III studies comparing Dara (intravenous or subcutaneous)-Pd with Pd (APOLLO, NCT 03180736), Dara-Kd with Kd (CANDOR, NCT 03158688), and Dara intravenous versus subcutaneous administrations (COLUMBA, NCT 03277105) 
are currently ongoing.

Elotuzumab:Elotuzumab is a $\mathrm{mAb}$ that targets signaling lymphocytic activation molecule F7 (SLAMF-7) on multiple myeloma cells. In a phase 1 trial, elotuzumab as a single agent showed little clinical efficacy [61]. However, elotuzumab combined with Rd showed significantly better efficacy, which was confirmed in the phase III ELOQUENT-2 trial. Elotuzumab plus Rd showed significantly better efficacy in terms of ORR (79\% vs. 66\%) and PFS (19.4 vs. 14.9 mo). Moreover, a better PFS was observed regardless of age group and renal failure [22]. Response was maintained even at 5 -year follow-up [62]. Common adverse events were hematologic toxicity and fatigue, which were similar in both treatment groups, and IRR occurred in $10 \%$ of patients in the elotuzumab arm who were mostly categorized as either grade 1 or 2. Phase II studies on the efficacy of elotuzumab with $\mathrm{Pd}$ or $\mathrm{Kd}$ are currently ongoing.

Conventional agents: Dexamethasone is used as a backbone for almost all the combination therapies at a weekly dose of $40 \mathrm{mg}$ or $20 \mathrm{mg}$, or even less for elderly, fragile patients. Corticosteroid use (dexamethasone weekly or prednisone $20 \mathrm{mg}$ daily) can be considered for patients who have exhausted all available options, with a caution to infections. Cyclophosphamide is often incorporated into combination therapy with bortezomib [63] or pomalidomide [49] and has recently shown efficacy with ixazomib [64]. Cyclophosphamide as a monotherapy or with dexamethasone can be administered via an oral or intravenous route, according to various doses and schedules. High-dose cyclophosphamide (600 mg/m $\mathrm{m}^{2}$ for 4 days) was found to have an ORR of $43 \%$ in advanced refractory $\mathrm{MM}$ in the period when no novel agents were available [65]. Melphalan is an historical remedy for $\mathrm{MM}$, and is still used as a drug for conditioning therapy during autoSCT and in combination with bortezomib or thalidomide. It could be an alternative for non-affordable new agents or for those who have no other therapeutic options. In certain circumstances such as the aggressive progression of MM, multidrug combination chemotherapy may be needed such as dexamethasone, cyclophosphamide, etoposide, and cisplatin (DCEP) [66]; thalidomide, dexamethasone, cisplatin, doxorubicin, cyclophosphamide, and etoposide (TD-PACE) [67]; or bortezomib, thalidomide, dexamethasone, cisplatin, doxorubicin, cyclophosphamide, and etoposide (VTD-PACE) [68] for rapid and effective control of the disease.

Bendamustine: Bendamustine, a nitrogen mustard derivative bifunctional alkylating agent, has been approved for use in transplant-ineligible newly diagnosed patients with MM who are not able to tolerate thalidomide or bortezomib due to severe peripheral neuropathy at presentation. Nowadays, its use has been reported to be more frequent in relapsed and refractory cases [69]. A dose-escalation trial involving refractory patients with $\mathrm{MM}$ after autoSCT reported an ORR of $55 \%$ and a median PFS of 8.5 months. From a real-world analysis in Korea, heavily pretreated patients with MM (median prior treatment, 5) who had been treated with bendamustine $\left(120 \mathrm{mg} / \mathrm{m}^{2}\right.$ on days 1 and 2$)$ plus prednisone showed an ORR of $35 \%$ and a median OS of 5.5 months [70]. Bendamustine in combination with lenalidomide [71] and bortezomib [72] has also shown activity in patients with RRMM.

Other food and drug administration (FDA)-approved drugs

Panobinostat: Panobinostat, a pan-histone deacetylase inhibitor, in combination with Vd showed an improved PFS (11.99 vs. $8.08 \mathrm{mo}$ ) and an nCR or better rate of $27.6 \%$ versus $15.7 \%$ but not in terms of the ORR $(60.7 \%$ vs. $54.6 \%)$ and OS (33.64 vs. $30.39 \mathrm{mo}$ ) compared with the control $\mathrm{Vd}$ arm in the phase III PANORAMA-1 study [73]. The major adverse events from the addition of panobinostat were cytopenia, diarrhea, and asthenia. Panobinostat has been approved by the US FDA for the treatment of patients with RRMM who failed to respond to bortezomib and lenalidomide; however, it has not been approved for use in Korea.

Selinexor:Selinexor, a selective inhibitor of exportin 1 (XPO1), has demonstrated its efficacy in triple-class refractory (at least one PI, one IMiD, and Dara) patients with MM [74] and has recently been approved by the US FDA. In highly refractory patients who were treated with a median of 7 regimens, the ORR was $26 \%$ with a median PFS and OS of 3.7 months and 8.6 months, respectively. Due to the promising results from the phase II clinical trial that evaluated selinexor plus Vd, a phase III trial comparing Vd with or without selinexor is currently underway (BOSTON trial, NCT03110562).

Novel therapies under investigation: There are several anti-CD38 mAbs, such as isatuximab and MOR202, that are undergoing clinical trials. One next-generation alkylating agent, melflufen, is expected to have a positive result in the phase III confirmatory trial. Novel drugs with different targets, such as anti-CD138 mAb-drug conjugate (indatuximab ravtansine), anti-PD-1 mAb (pembrolizumab), and oral bcl-2 inhibitor (venetoclax) are being actively examined. Novel treatment strategies such as bispecific T-cell engager antibodies, cyclin-dependent kinase inhibitors, kinesin spindle protein inhibitors, oral HDAC6 inhibitors, and chimeric antigen receptor (CAR) T-cell therapy are currently undergoing clinical investigation.

\section{Salvage transplantation}

A second autoSCT with high-dose therapy may be an option in transplant-eligible patients if sufficient stem cells have been collected [75]. Previous retrospective analyses have shown patients who relapsed $>18-24$ months after the first autoSCT may benefit from autoSCT after re-induction therapy $[76,77]$. Matched-pair analysis from the Korean myeloma registry has reported significantly longer OS (55.5 vs. $25.4 \mathrm{mo}$ ) for salvage autoSCT compared with systemic chemotherapy alone in patients who relapsed after upfront autoSCT [78]. A poor outcome could be predicted for patients who relapsed $<18$ months after their first autoSCT and ISS III. The only phase III German trial comparing re-induction Rd followed by salvage autoSCT and maintenance $\mathrm{Rd}$ with continuous $\mathrm{Rd}$ did not prove the efficacy 
of autoSCT as a salvage treatment in terms of ORR, PFS, and OS; however, patients with high-risk cytogenetics experienced improved survival after salvage autoSCT (HR, 2.71 for PFS and 4.22 for OS; $P<0.001$ for both) [79]. The role of salvage autoSCT is not clear in the era of frontline maintenance therapy and new drug triplet combinations in the RRMM setting. AlloSCT with reduced induced conditioning chemotherapy can be considered in young and fit patients who have high-risk cytogenetics. The largest outcome series, from European Group for Blood and Marrow Transplantation data, has reported a 3 -year PFS of $41 \%$ and OS of $21 \%$ in the heterogeneous population of RRMM [80]. Recent registry-based analysis comparing salvage autoSCT and alloSCT in patients with RRMM showed no significant advantage in terms of alloSCT in this setting; however, long-term survivors were only observed among alloSCT recipients [81].

\section{TREATMENT STRATEGY FOR RRMM}

Although no established guideline exists for the most optimal treatment of RRMM, practical treatment algorithms based on currently available study results and from Korean settings can be suggested (Fig. 1). Biochemical relapse not fulfilling the IMWG criteria for progressive disease may be followed up every 2 months, with close monitoring for the appearance of myeloma-related symptoms. Biochemical relapse that has met the IMWG criteria and clinical relapses should proceed to treatment. Aggressive biology (rapid onset of hypercalcemia, renal insufficiency and skeletal events, newly appearing extramedullary plasmacytoma, doubling of M-protein concentration, elevated LDH levels, elevated peripheral blood plasma cell counts, and a high plasma cell proliferation index) may be better treated with at least triplet combinations of novel agents, which are able to elicit a rapid and deep response. Patients in deep and durable responses, such as those with a response duration of $>24$ months to frontline therapy, from 18 to 24 months after autoSCT without maintenance, and from 36 to 48 months after autoSCT with maintenance, can be retreated with previously prescribed drugs or undergo salvage HDT with autologous stem cell support [4]. Drugs that were previously refractory should be avoided. Patients refractory to bortezomib should receive lenalidomide-backbone triplets or doublets

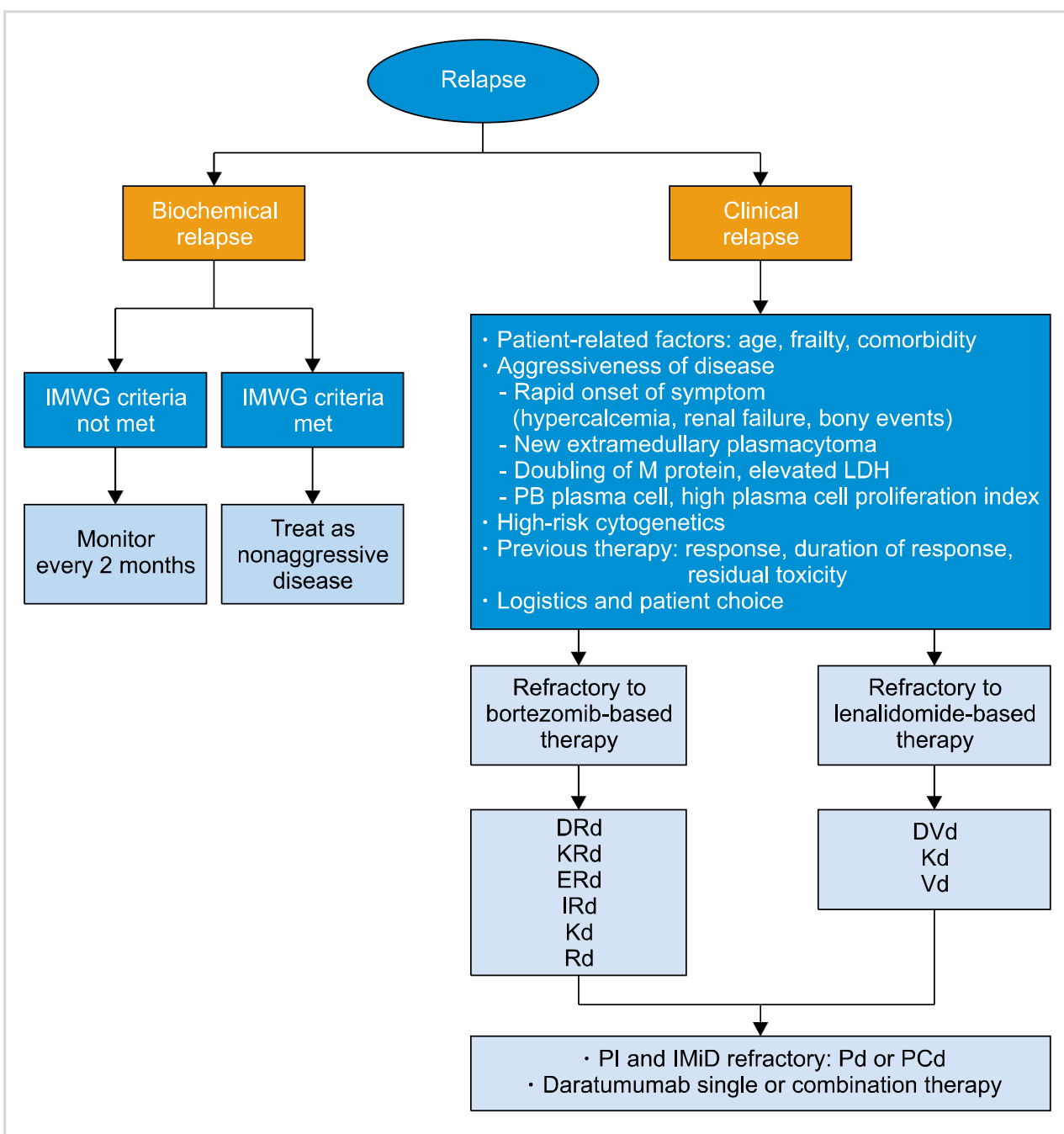

Fig. 1. Proposed treatment algorithm in relapsed and refractory multiple myeloma patients. Abbreviations: DRd, daratumumab$\mathrm{Rd}$; DVd, daratumumab-Vd; ERd, elotuzumab-Rd; IMiD, immunomodulatory drug; IMWG, international myeloma working group; IRd, ixazomib-Rd; $\mathrm{Kd}$, carfilzomibdexamethasone; KRd, carfilzomib$\mathrm{Rd}$; $\mathrm{LDH}$, lactate dehydrogenase; M protein, monoclonal protein; PCd, pomalidomide-cyclophosphamide, dexamethasone; Pd, pomalidomide, dexamethasone; $\mathrm{PI}$, proteasome inhibitor; Rd, lenalidomide-low-dose dexamethasone; $\mathrm{Vd}$, bortezomibdexamethasone. 
(KRd, IRd, DRd, Elo-Rd, and Rd) or Kd, while lenalidomide-refractory patients should be treated with $\mathrm{Kd}$ or $\mathrm{Vd}$ with or without daratumumab. Double-refractory patients to IMiDs and PIs can benefit from pomalidomide-based doublet or triplet therapies (Pd or PCd) and daratumumab monotherapy.

\section{CONCLUSION AND FUTURE PERSPECTIVES}

Owing to the recent approval of new agents by the Health Insurance Review \& Assessment (HIRA) Service in the Republic of Korea, the outcome concerning MM has markedly improved. Real-world clinical experience using the approved drugs and their combinations in Korea have shown similar efficacy and toxicity profiles compared with results from the clinical trials. Well-organized long-term observational studies are needed to confirm the beneficial effect of novel agents, and efforts to develop newer treatment modalities are required to prolong survival for patients with MM.

\section{Authors' Disclosures of Potential Conflicts of Interest}

No potential conflicts of interest relevant to this article were reported.

\section{REFERENCES}

1. Kumar SK, Dimopoulos MA, Kastritis E, et al. Natural history of relapsed myeloma, refractory to immunomodulatory drugs and proteasome inhibitors: a multicenter IMWG study. Leukemia 2017;31:2443-8.

2. Anderson KC, Kyle RA, Rajkumar SV, et al. Clinically relevant end points and new drug approvals for myeloma. Leukemia 2008;22:231-9.

3. Kumar S, Paiva B, Anderson KC, et al. International Myeloma Working Group consensus criteria for response and minimal residual disease assessment in multiple myeloma. Lancet Oncol 2016;17:e328-46.

4. Laubach J, Garderet L, Mahindra A, et al. Management of relapsed multiple myeloma: recommendations of the International Myeloma Working Group. Leukemia 2016;30:1005-17.

5. Sonneveld P. Management of multiple myeloma in the relapsed/ refractory patient. Hematology Am Soc Hematol Educ Program 2017;2017:508-17.

6. Kumar SK, Dispenzieri A, Lacy MQ et al. Continued improvement in survival in multiple myeloma: changes in early mortality and outcomes in older patients. Leukemia 2014;28: 1122-8.

7. Salazar AS, Recinos LM, Mian HS, et al. Geriatric assessment and frailty scores predict mortality in myeloma: systematic review and meta-analysis. Clin Lymphoma Myeloma Leuk 2019;19:488-96, e6.

8. Palumbo A, Bringhen S, Mateos MV, et al. Geriatric assessment predicts survival and toxicities in elderly myeloma patients: an International Myeloma Working Group report. Blood 2015;125: 2068-74.

9. Lee JH, Kim SH, Huh SJ, et al. The survival of transplant ineligible multiple myeloma patients treated with bortezomib-based chemotherapy is affected by the patient fitness. Blood (ASH Annual Meeting Abstracts) 2018;132(Suppl):3307.

10. Lenhoff $S$, Hjorth M, Turesson I, et al. Intensive therapy for multiple myeloma in patients younger than 60 years. Long-term results focusing on the effect of the degree of response on survival and relapse pattern after transplantation. Haematologica 2006;91:1228-33.

11. Rasche L, Bernard C, Topp MS, et al. Features of extramedullary myeloma relapse: high proliferation, minimal marrow involvement, adverse cytogenetics: a retrospective single-center study of 24 cases. Ann Hematol 2012;91:1031-7.

12. Lee H, Duggan P, Chaudhry A, et al. Early relapse for multiple myeloma patients undergoing single autologous stem cell therapy: a single-center experience. Clin Lymphoma Myeloma Leuk 2018;18:e69-75.

13. Gonsalves WI, Morice WG, Rajkumar V, et al. Quantification of clonal circulating plasma cells in relapsed multiple myeloma. $\mathrm{Br}$ J Haematol 2014;167:500-5.

14. Sidiqi MH, Aljama MA, Jevremovic D, et al. Plasma cell proliferative index post-transplant is a powerful predictor of prognosis in myeloma patients failing to achieve a complete response. Bone Marrow Transplant 2019;54:442-7.

15. Offidani M, Boccadoro M, Di Raimondo F, Petrucci MT, Tosi P, Cavo M. Expert panel consensus statement for proper evaluation of first relapse in multiple myeloma. Curr Hematol Malig Rep 2019;14:187-96.

16. Avet-Loiseau H, Soulier J, Fermand JP, et al. Impact of high-risk cytogenetics and prior therapy on outcomes in patients with advanced relapsed or refractory multiple myeloma treated with lenalidomide plus dexaméthasone. Leukemia 2010;24:623-8.

17. Reece D, Song KW, Fu T, et al. Influence of cytogenetics in patients with relapsed or refractory multiple myeloma treated with lenalidomide plus dexamethasone: adverse effect of deletion 17p13. Blood 2009;114:522-5.

18. Chng WJ, Goldschmidt H, Dimopoulos MA, et al. Carfilzomibdexamethasone vs bortezomib-dexamethasone in relapsed or refractory multiple myeloma by cytogenetic risk in the phase 3 study ENDEAVOR. Leukemia 2017;31:1368-74.

19. Moreau P, Masszi T, Grzasko N, et al. Oral ixazomib, lenalidomide, and dexamethasone for multiple myeloma. N Engl J Med 2016;374:1621-34.

20. Palumbo A, Chanan-Khan A, Weisel K, et al. Daratumumab, bortezomib, and dexamethasone for multiple myeloma. N Engl J Med 2016;375:754-66.

21. Avet-Loiseau H, Fonseca R, Siegel D, et al. Carfilzomib significantly improves the progression-free survival of high-risk patients in multiple myeloma. Blood 2016;128:1174-80.

22. Lonial S, Dimopoulos M, Palumbo A, et al. Elotuzumab therapy for relapsed or refractory multiple myeloma. N Engl J Med 2015;373:621-31.

23. Dimopoulos MA, Oriol A, Nahi H, et al. Daratumumab, lenalidomide, and dexamethasone for multiple myeloma. $\mathrm{N}$ Engl 
J Med 2016;375:1319-31.

24. Moreau P, Siegel DS, Goldschmidt H, et al. Subgroup analysis of patients with biochemical or symptomatic relapse at the time of enrollment in the endeavor study. Blood (ASH Annual Meeting Abstracts) 2018;132(Suppl):3243.

25. Hulin C, de la Rubia J, Dimopoulos MA, et al. Bortezomib retreatment for relapsed and refractory multiple myeloma in real-world clinical practice. Health Sci Rep 2018;2:e104.

26. Madan S, Lacy MQ Dispenzieri A, et al. Efficacy of retreatment with immunomodulatory drugs (IMiDs) in patients receiving IMiDs for initial therapy of newly diagnosed multiple myeloma. Blood 2011;118:1763-5.

27. Richardson PG, Sonneveld P, Schuster MW, et al. Bortezomib or high-dose dexamethasone for relapsed multiple myeloma. $\mathrm{N}$ Engl J Med 2005;352:2487-98.

28. Koh Y, Lee SY, Kim I, et al. Bortezomib-associated peripheral neuropathy requiring medical treatment is decreased by administering the medication by subcutaneous injection in Korean multiple myeloma patients. Cancer Chemother Pharmacol 2014; 74:653-7.

29. Palumbo A, Bringhen S, Ludwig H, et al. Personalized therapy in multiple myeloma according to patient age and vulnerability: a report of the European Myeloma Network (EMN). Blood 2011; 118:4519-29.

30. Orlowski RZ, Nagler A, Sonneveld P, et al. Randomized phase III study of pegylated liposomal doxorubicin plus bortezomib compared with bortezomib alone in relapsed or refractory multiple myeloma: combination therapy improves time to progression. J Clin Oncol 2007;25:3892-901.

31. Garderet L, Iacobelli S, Moreau P, et al. Superiority of the triple combination of bortezomib-thalidomide-dexamethasone over the dual combination of thalidomide-dexamethasone in patients with multiple myeloma progressing or relapsing after autologous transplantation: the MMVAR/IFM 2005-04 randomized phase III trial from the Chronic Leukemia Working Party of the European Group for Blood and Marrow Transplantation. J Clin Oncol 2012;30:2475-82.

32. Hájek R, Masszi T, Petrucci MT, et al. A randomized phase III study of carfilzomib vs low-dose corticosteroids with optional cyclophosphamide in relapsed and refractory multiple myeloma (FOCUS). Leukemia 2017;31:107-14.

33. Stewart AK, Rajkumar SV, Dimopoulos MA, et al. Carfilzomib, lenalidomide, and dexamethasone for relapsed multiple myeloma. N Engl J Med 2015;372:142-52.

34. Siegel DS, Dimopoulos MA, Ludwig H, et al. Improvement in overall survival with carfilzomib, lenalidomide, and dexamethasone in patients with relapsed or refractory multiple myeloma. J Clin Oncol 2018;36:728-34.

35. Dimopoulos MA, Stewart AK, Masszi T, et al. Carfilzomib, lenalidomide, and dexamethasone in patients with relapsed multiple myeloma categorised by age: secondary analysis from the phase 3 ASPIRE study. Br J Haematol 2017;177:404-13.

36. Dimopoulos MA, Moreau P, Palumbo A, et al. Carfilzomib and dexamethasone versus bortezomib and dexamethasone for patients with relapsed or refractory multiple myeloma (ENDEAVOR): a randomised, phase 3, open-label, multicentre study. Lancet Oncol 2016;17:27-38.
37. Moreau P, Mateos MV, Berenson JR, et al. Once weekly versus twice weekly carfilzomib dosing in patients with relapsed and refractory multiple myeloma (A.R.R.O.W.): interim analysis results of a randomised, phase 3 study. Lancet Oncol 2018;19: 953-64.

38. Dimopoulos MA, Moreau P, Iida S, et al. Outcomes for Asian patients with multiple myeloma receiving once- or twice-weekly carfilzomib-based therapy: a subgroup analysis of the randomized phase 3 ENDEAVOR and A.R.R.O.W. Trials. Int J Hematol 2019;110:466-73.

39. Ghobrial IM, Vij R, Siegel D, et al. A phase Ib/II study of oprozomib in patients with advanced multiple myeloma and Waldenström macroglobulinemia. Clin Cancer Res 2019;25:4907-16.

40. Shah J, Usmani S, Stadtmauer EA, et al. Oprozomib, pomalidomide, and dexamethasone in patients with relapsed and/or refractory multiple myeloma. Clin Lymphoma Myeloma Leuk 2019;19: 570-8, e1.

41. Harrison SJ, Mainwaring P, Price T, et al. Phase I clinical trial of marizomib (NPI-0052) in patients with advanced malignancies including multiple myeloma: study NPI-0052-102 final results. Clin Cancer Res 2016;22:4559-66.

42. Kwon J, Min CK, Kim K, et al. Efficacy and toxicity of the combination chemotherapy of thalidomide, alkylating agent, and steroid for relapsed/refractory myeloma patients: a report from the Korean Multiple Myeloma Working Party (KMMWP) retrospective study. Cancer Med 2017;6:100-8.

43. Dimopoulos MA, Chen C, Spencer A, et al. Long-term follow-up on overall survival from the MM-009 and MM-010 phase III trials of lenalidomide plus dexamethasone in patients with relapsed or refractory multiple myeloma. Leukemia 2009;23:2147-52.

44. Dimopoulos MA, Palumbo A, Attal M, et al. Optimizing the use of lenalidomide in relapsed or refractory multiple myeloma: consensus statement. Leukemia 2011;25:749-60.

45. Kim K, Kim SJ, Voelter V, et al. Lenalidomide with dexamethasone treatment for relapsed/refractory myeloma patients in Koreaexperience from 110 patients. Ann Hematol 2014;93:113-21.

46. Richardson PG, Xie W, Jagannath S, et al. A phase 2 trial of lenalidomide, bortezomib, and dexamethasone in patients with relapsed and relapsed/refractory myeloma. Blood 2014;123: 1461-9.

47. Miguel JS, Weisel K, Moreau P, et al. Pomalidomide plus low-dose dexamethasone versus high-dose dexamethasone alone for patients with relapsed and refractory multiple myeloma (MM-003): a randomised, open-label, phase 3 trial. Lancet Oncol 2013;14:1055-66.

48. Leleu X, Karlin L, Macro M, et al. Pomalidomide plus low-dose dexamethasone in multiple myeloma with deletion $17 \mathrm{p}$ and/or translocation (4;14): IFM 2010-02 trial results. Blood 2015;125: 1411-7.

49. Baz RC, Martin TG 3rd, Lin HY, et al. Randomized multicenter phase 2 study of pomalidomide, cyclophosphamide, and dexamethasone in relapsed refractory myeloma. Blood 2016;127:2561-8.

50. Lee JH, Kim SH, Lee YJ, et al. The comparison of carfilzomib, and dexamethasone versus pomalidomide-based combination chemotherapy after a 2nd-line therapy in relapsed and refractory multiple myeloma patients. Clin Lymphoma Myeloma Leuk (International Myeloma Workshop Abstracts) 2019;19(Suppl): 
e264-5.

51. Richardson PG, Oriol A, Beksac M, et al. Pomalidomide, bortezomib, and dexamethasone for patients with relapsed or refractory multiple myeloma previously treated with lenalidomide (OPTIMISMM): a randomised, open-label, phase 3 trial. Lancet Oncol 2019;20:781-94.

52. Lokhorst HM, Plesner T, Laubach JP, et al. Targeting CD38 with daratumumab monotherapy in multiple myeloma. $\mathrm{N}$ Engl J Med 2015;373:1207-19.

53. Lonial S, Weiss BM, Usmani SZ, et al. Daratumumab monotherapy in patients with treatment-refractory multiple myeloma (SIRIUS): an open-label, randomised, phase 2 trial. Lancet 2016;387: 1551-60.

54. Usmani SZ, Weiss BM, Plesner T, et al. Clinical efficacy of daratumumab monotherapy in patients with heavily pretreated relapsed or refractory multiple myeloma. Blood 2016;128:37-44.

55. Usmani SZ, Nahi H, Weiss BM, et al. Safety and efficacy of daratumumab monotherapy in patients with heavily pretreated relapsed and refractory multiple myeloma: final results from GEN501 and SIRIUS. Blood (ASH Annual Meeting Abstracts) 2017;130(Suppl):3107.

56. Park SS, Eom HS, Kim JS, et al. Brief report: clinical experiences after emergency use of daratumumab monotherapy for relapsed or refractory multiple myeloma in real practice. Jpn J Clin Oncol 2019;49:92-5.

57. Byun JM, Yoon SS, Koh Y, et al. Daratumumab monotherapy in heavily pretreated Asian patients with relapsed and refractory multiple myeloma: a real-world experience. Anticancer Res 2019;39:5165-70.

58. Dimopoulos MA, San-Miguel J, Belch A, et al. Daratumumab plus lenalidomide and dexamethasone versus lenalidomide and dexamethasone in relapsed or refractory multiple myeloma: updated analysis of POLLUX. Haematologica 2018;103:2088-96.

59. Spencer A, Lentzsch S, Weisel K, et al. Daratumumab plus bortezomib and dexamethasone versus bortezomib and dexamethasone in relapsed or refractory multiple myeloma: updated analysis of CASTOR. Haematologica 2018;103:2079-87.

60. San-Miguel J, Weisel K, Cook G, et al. Efficacy by cytogenetic risk status for daratumumab in combination with lenalidomide and dexamethasone or bortezomib and dexamethasone in relapsed or refractory multiple myeloma. Haematologica 2017;102:1-2.

61. Zonder JA, Mohrbacher AF, Singhal S, et al. A phase 1, multicenter, open-label, dose escalation study of elotuzumab in patients with advanced multiple myeloma. Blood 2012;120: 552-9.

62. Lonial S, Dimopoulos MA, Weisel K, et al. Extended 5-y follow-up (FU) of phase 3 ELOQUENT-2 study of elotuzumab+ lenalidomide/ dexamethasone (ELd) vs Ld in relapsed/refractory multiple myeloma (RRMM). J Clin Oncol (ASCO Annual Meeting Abstracts) 2018;36(Suppl):8040.

63. Davies FE, Wu P, Jenner M, Srikanth M, Saso R, Morgan GJ. The combination of cyclophosphamide, velcade and dexamethasone induces high response rates with comparable toxicity to velcade alone and velcade plus dexamethasone. Haematologica 2007;92: 1149-50.

64. Kumar SK, Grzasko N, Delimpasi S, et al. Phase 2 study of all-oral ixazomib, cyclophosphamide and low-dose dexamethasone for relapsed/refractory multiple myeloma. Br J Haematol 2019;184: 536-46.

65. Case DC Jr, Young CW, Lee BJ 3rd. Combination chemotherapy of MOPP-resistant Hodgkin's disease with adriamycin, bleomycin, dacarbazine and vinblastine (ABDV). Cancer 1977;39:1382-6.

66. Park S, Lee SJ, Jung CW, et al. DCEP for relapsed or refractory multiple myeloma after therapy with novel agents. Ann Hematol 2014;93:99-105.

67. Lee CK, Barlogie B, Munshi N, et al. DTPACE: an effective, novel combination chemotherapy with thalidomide for previously treated patients with myeloma. J Clin Oncol 2003;21:2732-9.

68. Lakshman A, Singh PP, Rajkumar SV, et al. Efficacy of VDT PACE-like regimens in treatment of relapsed/refractory multiple myeloma. Am J Hematol 2018;93:179-86.

69. Knop S, Straka C, Haen M, Schwedes R, Hebart H, Einsele H. The efficacy and toxicity of bendamustine in recurrent multiple myeloma after high-dose chemotherapy. Haematologica 2005;90: 1287-8.

70. Kim SJ, Bang SM, Choi YS, et al. Bendamustine in heavily pre-treated multiple myeloma patients: results of a retrospective analysis from the Korean Multiple Myeloma Working Party. Blood Res 2016;51:193-9.

71. Lentzsch S, O'Sullivan A, Kennedy RC, et al. Combination of bendamustine, lenalidomide, and dexamethasone (BLD) in patients with relapsed or refractory multiple myeloma is feasible and highly effective: results of phase $1 / 2$ open-label, dose escalation study. Blood 2012;119:4608-13.

72. Offidani M, Corvatta L, Maracci L, et al. Efficacy and tolerability of bendamustine, bortezomib and dexamethasone in patients with relapsed-refractory multiple myeloma: a phase II study. Blood Cancer J 2013;3:e162.

73. San-Miguel JF, Hungria VT, Yoon SS, et al. Panobinostat plus bortezomib and dexamethasone versus placebo plus bortezomib and dexamethasone in patients with relapsed or relapsed and refractory multiple myeloma: a multicentre, randomised, doubleblind phase 3 trial. Lancet Oncol 2014;15:1195-206.

74. Chari A, Vogl DT, Gavriatopoulou M, et al. Oral selinexordexamethasone for triple-class refractory multiple myeloma. $\mathrm{N}$ Engl J Med 2019;381:727-38.

75. Giralt S, Garderet L, Durie B, et al. American Society of Blood and Marrow Transplantation, European Society of Blood and Marrow Transplantation, Blood and Marrow Transplant Clinical Trials Network, and International Myeloma Working Group Consensus Conference on Salvage Hematopoietic Cell Transplantation in Patients with Relapsed Multiple Myeloma. Biol Blood Marrow Transplant 2015;21:2039-51.

76. Sellner L, Heiss C, Benner A, et al. Autologous retransplantation for patients with recurrent multiple myeloma: a single-center experience with 200 patients. Cancer 2013;119:2438-46.

77. Cook G, Ashcroft AJ, Cairns DA, et al. The effect of salvage autologous stem-cell transplantation on overall survival in patients with relapsed multiple myeloma (final results from BSBMT/UKMF Myeloma X Relapse [Intensive]): a randomised, open-label, phase 3 trial. Lancet Haematol 2016;3:e340-51.

78. Yhim HY, Kim K, Kim JS, et al. Matched-pair analysis to compare the outcomes of a second salvage auto-SCT to systemic chemotherapy alone in patients with multiple myeloma who 
relapsed after front-line auto-SCT. Bone Marrow Transplant 2013;48:425-32.

79. Goldschmidt H, Baertsch MA, Schlenzka J, et al. Salvage autologous transplant and lenalidomide maintenance versus continuous lenalidomide/dexamethasone for relapsed multiple myeloma: results of the randomized GMMG phase III multicenter trial relapse. Blood (ASH Annual Meeting Abstracts) 2018; 132(Suppl):253.
80. Crawley C, Lalancette M, Szydlo R, et al. Outcomes for reduced-intensity allogeneic transplantation for multiple myeloma: an analysis of prognostic factors from the Chronic Leukaemia Working Party of the EBMT. Blood 2005;105:4532-9.

81. Ikeda T, Mori K, Kawamura K, et al. Comparison between autologous and allogeneic stem cell transplantation as salvage therapy for multiple myeloma relapsing/progressing after autologous stem cell transplantation. Hematol Oncol 2019;37:586-94. 